De quelques enjeux et usages historiques du Français fondamental

\title{
Le Français fondamental et les vocabulaires de base en Allemagne
}

Franz-Joseph Meissner

\section{(2) OpenEdition Journals}

Édition électronique

URL : https://journals.openedition.org/dhfles/1198

DOI : $10.4000 /$ dhfles. 1198

ISSN : 2221-4038

Éditeur

Société Internationale pour l'Histoire du Français Langue Étrangère ou Seconde

Édition imprimée

Date de publication : 1 janvier 2006

Pagination : 119-137

ISSN : 0992-7654

Référence électronique

Franz-Joseph Meissner, "Le Français fondamental et les vocabulaires de base en Allemagne », Documents pour l'histoire du français langue étrangère ou seconde [En ligne], 36 | 2006, mis en ligne le 06 septembre 2011, consulté le 27 mai 2021. URL : http://journals.openedition.org/dhfles/1198 ; DOI : https://doi.org/10.4000/dhfles.1198

Ce document a été généré automatiquement le 27 mai 2021

(c) SIHFLES 


\title{
Le Français fondamental et les vocabulaires de base en Allemagne
}

\author{
Franz-Joseph Meissner
}

1 Le rôle joué par le Français fondamental (dorénavant FF) outre-Rhin est largement tributaire des circonstances pédagogiques, socioculturelles et politiques des années qui précèdent la confection du Français élémentaire (FE), en 1954, voire du FF en 1958 pour la première édition. Pendant les années 50 , les articles des revues didactiques allemandes tournent autour de deux pôles : le souhait de rendre l'enseignement plus efficace et une pratique scolaire encore et toujours dans le sillage d'une méthodologie telle qu'on l'avait connue avant la guerre, orientée plutôt sur les habilités à lire et à écrire que sur la compétence communicative orale. Les glossaires pédagogiques de base ne se trouvent pas au centre des questions traitées dans ces revues.

\section{Quelques remarques préliminaires}

2 Dès son origine, le discours français menant à la composition du FF dépasse les sphères de la didactique des langues au sens strict car il se déroule à un moment où anglophones et francophones se proposent de partager le monde. Faisant écho à une interview avec le linguiste A. Sauvageot, publiée dans le Figaro Littéraire du 5 janvier 1952, le public averti allemand pouvait replacer l'origine du FF dans un colloque organisé par l'UNESCO à Paris en 1947. On y avait discuté les possibilités de faciliter l'acquisition de connaissances opérables dans au moins une langue prestigieuse de culture à un maximum de personnes. Un accord plus ou moins tacite voulait que les langues en question fussent exclusivement l'anglais et le français. «M. Sauvageot (...) obtint que le français fût adopté en Europe et dans la majeure partie de l'Afrique. Il serait également admis en concurrence en Amérique du Sud, dans le Moyen et l'Extrême Orient. Partout ailleurs, l'anglais prévaudrait ", lisait-on dans Le Monde le 1er novembre 1952. En même temps, le Colloque prévoyait un soutien des langues régionales. Pour réaliser le projet, l'UNESCO souhaitait la confection d'un français de base, tâche qui fût assez vite confiée au Ministère de l'Éducation français. 
$3 \mathrm{Au}$ moment où l'UNESCO prit cette décision, les vocabulaires de base avaient déjà fait preuve d'une remarquable utilité grâce au BASIC-English de C. K. Ogden. Comme l'indique l'acronyme - British American Scientific International Commercial -, il s'agissait d'une sorte de registre linguistique artificiel dont la maîtrise devait permettre la communication fonctionnelle anglophone « world and wide ».

Il va sans dire que les apprenants pouvaient toujours aller plus loin, vers une compétence plus parfaite. Le fait que le BASIC passait pour étonnamment efficace, explique le soutien que lui a accordé le gouvernement britannique pendant longtemps. H. Zeidler (1980: 7) observe : «Dans plus de vingt pays, on apprenait l'anglais à l'aide du BASIC. En outre, plus de 20.000 matelots chinois qui servaient dans la marine américaine, avaient appris le BASIC en seulement trois mois : la maîtrise de 500 mots de la vie quotidienne et de 350 termes militaires leur permettaient de s'engager dans la guerre aux côtés des Anglais. Le colonel anglais Myers avait réussi à enseigner le BASIC à 2.000.000 Indiens en six mois... $»^{1}$ - Dans les discussions allemandes postérieures, on ne semblait pas souvent avoir compris la dimension politique du BASIC. Les confusions étaient évidemment dues à l'homophonie entre l'acronyme et l'épithète Basic 'basique, fondamental'.

5 Ce bref regard sur la situation qui est à l'origine du FF nous montre d'ores et déjà pourquoi, dans l'Allemagne de l'après-guerre et des années 50, les discussions précédant la fabrication du FF ont laissé peu de traces. D'autant que pour l'enseignement de l'anglais en RFA, le BASIC paraissait peu utile car les objectifs pédagogiques y continuaient à viser plutôt les compétences scripturales qu'orales.

\section{La genèse de l'intérêt pour le lexique de base de FLE en Allemagne}

6 Avant que le FE ne fût remplacé par le FF, E. P. Heibel à qui on en avait confié la traduction, observe en 1958 dans la préface de la brochure, publiée par l'Institut Français de Mayence et intitulée Français élémentaire. Deutsche Fassung der vom französischen Unterrichtsministerium 1954 herausgegebenen Broschüre über 'Elementarfranzösisch':

Das grosse Interesse, welches seit einiger Zeit Pädagogen und Wissenschaftler in Deutschland dem ,Français Élémentaire' entgegenbringen, veranlasst uns, die 1954 vom Französischen Unterrichtsministerium herausgegebene Broschüre in Übersetzung zu veröffentlichen, damit weite Kreise mit diesem für die erleichterte aber korrekte Erlernung der französischen Sprache so wichtigen Versuch vertraut gemacht werden können. (Ministère de l'Éducation Nationale $1958: 1$ )

7 Une courte bibliographie mentionne Le français élémentaire, méthode progressive de français parlé de $\mathrm{G}$. Mauger et $\mathrm{G}$. Gougenheim (Hachette) ainsi que le Premier dictionnaire en image, les 1.300 mots fondamentaux du français de P. Fourré (Didier) et renvoie à L'élaboration du français élémentaire. Etude sur l'établissement d'un vocabulaire et d'une grammaire de base de Gougenheim et al. (1956). Tout à fait dans le sillage de cet ouvrage, la brochure expose les problèmes ayant trait à la confection d'un vocabulaire de base destiné à l'apprentissage d'une langue étrangère : écarts entre les lexiques du français écrit et parlé, computation des mots des registres parlés, sélection des lemmes (fréquence, répartition, disponibilité) et présentation du lexique. Le FE se veut une liste 
ouverte et adaptable aux besoins différents de contextes d'apprentissage divers ${ }^{2}$. Dans l'avant-propos, on lit :

Das Elementarfranzösisch wurde für die umfassende und schnelle Verbreitung der französischen Sprache geschaffen. (...)

Es geht (...) darum, (...) dem Lehrer die unmittelbar nützlichen Begriffe und Mechanismen an die Hand zu geben, die 'Grundlagen', auf welcher er seinen Unterricht stützen muss, um die Einführung in die französische Sprache schneller und wirksamer zu gestalten.

\section{Le FF et les instructions des Länder}

Les observations sur le BASIC et le FE attirent notre attention sur les expériences allemandes de l'époque dans le domaine des vocabulaires de base. Selon les bibliographies, peu de glossaires de ce type avaient été confectionnés outre-Rhin avant 1960. Ceci s'explique par le fait déjà mentionné que les LV y étaient de fait encore et toujours enseignées à des fins scripturales. Tant que le traitement de textes écrits dominait les objectifs des LV, les vocabulaires de basses fréquences se révélaient peu utiles. Ainsi note L. Michelini (1988: 272) : « ... nella lingua parlata la ripetizione (che è quella che fornisce la frequenza delle parole) interviene prima (...): nella lingua scritta per avère questa ripetizione (...), si ha bisogno di un corpus molto più vasto ". Notons par ailleurs que le terme Hörverstehen (compréhension auditive, listening comprehension) n'apparait que peu avant 1970 dans les instructions allemandes (Meissner 2000), et que la linguistique descriptive du français parlé était encore dans les langes (Greive 1984). Quant au déploiement des compétences orales, les instructions bavaroises pour les LV qui remplacent, en 1964, les 'Stundentafeln und Stoffpläne für die Höheren Lehranstalten' de 1952 (Zapp et Schröder 1983 : 199) recommandent encore la lecture à haute voix, les conversations simples à partir des textes traités en classe, des résumés et de brefs comptes rendus de lecture. On n'y lit aucune référence à la compréhension auditive. La 'didactique communicative' n'avait apparemment pas encore atteint les auteurs des instructions scolaires bien que des programmes audiovisuels et les laboratoires des langues fussent de plus en plus disponibles. Comme les types d'examen influent largement sur les pratiques de l'enseignement, force est de souligner que le compte rendu de lecture (où l'enseignant lisait à haute voix un texte fort de 800 à 1.200 mots pour le faire reproduire sous forme écrite par les élèves) était la forme de test dominante de l'Abitur (baccalauréat) jusque dans les années 70.

Par conséquent, D. Lübke, auteur du Dictionnaire scolaire du français (1981: III), remarque dans l'avant-propos de son ouvrage que, hormis l'inventaire du FF, cette liste comprend le vocabulaire des manuels scolaires les plus usuels. Quelques années auparavant, cet auteur avait publié Emploi des mots. Lernwörterbuch in Sachgruppen qui incorpore les inventaires des FF1 et FF2 enrichis de 517 mots. Les lemmes y sont groupés par centres d'intérêt. Un livre d'exercices complète la liste du dictionnaire « d'apprentissage ». La pratique de Lübke reflète grosso modo la position des instructions scolaires allemandes. Quelques-unes d'entre elles recommandaient pour la Sekundarstufe II et les terminales le Vocabulaire général d'orientation scientifique d'A. Phal et al. (CREDIF 1971). Cela montre pourtant que l'idée d'orienter l'input lexical sur les listes bien définies était de rigueur.

10 Les instructions des Länder mentionnent le FF à partir de 1964, à commencer par la Bavière. Après deux années d'études de français, y lit-on, les élèves doivent disposer de 
1.600 à 2.000 mots dont les 1.300 enregistrements du FF1 (Bavière $1964: 381$; cité par Zeidler 1990 : 372).

11 Huit ans plus tard, les 'Rahmenrichtlinien Sekundarstufe I, Neue Sprachen' de la Hesse (1972 : 86/87) fourniront un bel exemple pour la présence du FF dans les instructions allemandes :

Mit dem Français Fondamental, 1er degré, (FF1), steht ein Instrumentarium zur Verfügung, das als Grundlage für die lernzielorientierte Auswahl von Lexik dienen kann. (...) Für die Auswahl des lexikalischen Materials in der Sekundarstufe I gelten daher folgende Grundsätze: 1. Aus der Liste, die sich bei der Frequenzuntersuchung zur Erstellung des FF1 ergab, werden - bis auf wenige Ausnahmen - alle jene Wörter übernommen, die auch in das FF1 Eingang gefunden haben. (...) Diese Wörter sind bei Französisch als 2. Fremdsprache am Ende der Sekundarstufe I aktiv zu beherrschen.

12 Tous les élèves hessois qui apprenaient le français, devaient donc disposer activement du répertoire FF à la fin du collège (15 à 16 ans). Le FF était devenu l'instrument par excellence pour la sélection du vocabulaire scolaire que les instructions des collèges mentionnent, à l'exception de celle de Brème, avec régularité.

La distinction du FF entre un premier et un second degré a sans doute contribué à une description échelonnée des niveaux de compétence ${ }^{3}$. Dans les écoles allemandes, cette différenciation se confondait avec le passage des élèves du collège (Sekundarstufe I), où le travail avec le manuel était prépondérant, au lycée (Sekundarstufe II), où des dossiers thématiques et des textes « authentiques » étoffaient les cours. G. Esser explique (1973 : 461) ce que tout cela signifiait au niveau de la pratique de l'enseignement du français dans la Sekundarstufe II. Le travail du lycée embrassait le vocabulaire du FF2 et un vocabulaire " écrit " destiné au traitement des questions abstraites telles qu'elles étaient prévues par l'Abitur.

La définition de deux ou trois niveaux de compétence coïncidait en plus avec la naissance de la 'Reformierte Gymnasiale Oberstufe' qui laissait aux élèves une certaine liberté de choisir leurs matières. Comme corollaire à cette individualisation, on a ressenti assez vite la nécessité de rendre les programmes plus transparents, d'où une tendance à décrire les objectifs et les méthodes pédagogiques de manière plus détaillée (voir : Franke 1977). Le FF apparaissait, là aussi, comme un outil auquel on avait recours régulièrement. Par conséquent, il ne surprend guère que, dans les années 70 , le FF devienne une référence régulière dans les instructions des ministères allemands de l'éducation.

L'importance de cette évolution ne saurait être surestimée car elle touchait, année par année, plus d'un million d'apprenants germanophones. Néanmoins, il y avait aussi des Länder qui préféraient des listes d'origine allemande au FF. Ainsi, les Instructions pour la Realschule de Hambourg (1974: 3) s'orientaient expressément sur le Vocabulaire minimal du français (titre original : Französischer Mindestwortschatz) d'A. Raasch de 1969. Ce glossaire est toutefois largement identique au FF1.

\section{Le FF et renseignement du français aux adultes}

La publicité du Französischer Mindestwortschatz publié aux éditions Max Hueber à Munich nous révèle une certaine confusion entre des intérêts commerciaux, des définitions d'objectifs pédagogiques et la discussion didactologique de l'époque. Ainsi, une 
annonce dans la revue Französisch an Volkshochschulen (1969: 12), prédécesseur de Zielsprache Französisch (à partir de 1972), souligne que ce glossaire « minimal » s'appuie sur le FF 1er et 2e degrés et des recherches entreprises par des experts allemands et français dont les résultats avaient été approuvés par le Comité chargé de définir un certificat de langue destiné aux universités populaires (VHS-Zertifikat). L'annonce suscite notre intérêt pour trois raisons. D'abord, elle prouve, tout comme les curricula scolaires, que le FF est, dans l'Allemagne de 1969, un facteur incontournable lorsqu'il est question de minima destinés à l'apprentissage du français. Deuxièmement, elle signale l'apparition d'un nouveau contexte d'apprentissage de plus en plus important : celui des LV à un public adulte ${ }^{4}$. En R.F.A., ce public passe très souvent par les universités populaires, ce qui explique le rôle que celles-ci prennent dans l'élaboration de nouveaux profils et certificats. Troisièmement, la certification indique une évolution qui dépasse clairement l'enseignement d'une langue-cible seule. Ainsi on assiste en très peu de temps à l'apparition d'autres VHS-Zertifikate pour l'espagnol en 1971 (A. Barrera-Vidal), pour l'italien en 1974 (H. Reiske)... et à la confection des vocabulaires et des grammaires de base confectionnés selon les objectifs stipulés par les certificats. Notons que Raasch était un des pionniers dans ce domaine. Il ne fait pas de doute que les certificats ont contribué aux succès des vocabulaires de base dont le FF.

Cet intérêt s'organisait en partie au sein du Fachverband Moderne Fremdsprachen dont le président F. J. Zapp, l'angliciste W. Hüllen et A. Raasch publient en 1977 une anthologie intitulée Sprachminima und Abschlußprofile. Elle dresse le bilan des théories des inventaires linguistiques en les combinant avec la certification. A cet ouvrage incombe le mérite d'avoir familiarisé un vaste public allemand spécialisé avec des questions ayant trait aux minima linguistiques. L'année 1972 voit aussi l'apparition d'une revue didactique destinée exclusivement à l'apprentissage des LV par les adultes, pour lequel la certification était primordiale : la Zielsprache Französisch (ZF). La thématique de la certification et des minima y est reprise avec une certaine régularité. En 1979, Le français dans le monde confie le cahier thématique L'enseignement du français aux adultes à Raasch, alors directeur de ZF. Tandis que S. Bolton et W. Halm (1979) décrivent le rôle du FF pour la composition du VHS-Zertifikat (Französisch) ${ }^{5}$, l'article de Raasch (1979b) montre que l'auto-évaluation fait alors partie d'une culture pédagogique en voie de renouvellement et dont les repères s'appelleront : centrage sur l'apprenant, évaluation, audits, modularisation de l'offre et capitalisation des acquis, apprentissage tout au long de la vie. Ces orientations augmentent, somme toute, la demande à disposer d'inventaires lexicaux et grammaticaux bien définis.

Les années 60 voient l'avènement des laboratoires des langues. En 1969, la revue Praxis des neusprachlichen Unterrichts présente quelques programmes pour le travail en LdL dont Voix et images de France (1er degré). Tous ces programmes portent sur le français parlé et incorporent, eux aussi, les structures du FF dont les succcès se confondent avec le renouvellement méthodologique de FLE.

Retenons qu'après 1975 au plus tard, le FF est présent dans tous les contextes ayant trait à l'apprentissage du français en Allemagne, scolaires et extra-scolaires, pratiques et théoriques (aspect dont on parlera plus tard). Cela va de pair avec la découverte de la compétence communicative et de l'oral au rang d'objectif pédagogique primordial. Le FF répond à ce moment-là à un intérêt qu'il a suscité en grande partie vingt ans plus tôt. Les vocabulaires de base plus ou moins " standardisés " sur des bases empiriques 
sont devenus un outil indispensable pour l'apprentissage des langues, tous contextes d'enseignement et langues-cible confondus.

\section{La réception du débat francophone autour du FF}

Il serait sans doute erroné de limiter l'influence du FF en Allemagne à l'ouvrage même. La réflexion critique que le $\mathrm{FF}$ suscite dans l'hexagone et au-delà se répercute aussi sur la théorie allemande des Grundwortschätze, et cette influence ne se limite pas à la Französischdidaktik.

21 Bien que l'Allemagne ait, depuis le dictionnaire de W. Kaeding (1897), développé des vocabulaires de base et qu'elle disposât d'une tradition lexicographique propre dans le domaine des minima ${ }^{6}$, les études reflètent, pendant la période considérée, l'influence du FF et des débats qu'il avait déclenchés. Dans ce contexte, on rappellera les publications de Michéa (1953), Gougenheim et al. (1964) qui retracent l'histoire antérieure au FF de cette lexicographie, Michéa (1969) à propos des fréquences et variétés des mots, de Savard et Richards (1970) sur les indices d'utilité du FF, Galisson $(1970,1971)$ critiquant l'ordre alphabétique du FF, l'absence du principe collocatif ou co-occurrentiel et d'une liste indicative des situations de communication, Michéa (1978), Rivenc (1979), Besse (1979). Ceci pris en compte, on retiendra que la revue Le français dans le monde a joué un rôle essentiel pour la propagation des idées lexicodidactiques de provenances française et québécoise non seulement en Allemagne, mais probablement dans le monde entier. L'énumération des chercheurs, liste à laquelle on pourrait facilement ajouter d'autres noms, documente des étapes importantes dans l'évolution de la théorie francophone sur les vocabulaires élémentaires. Tandis que les premiers articles expliquaient encore les avantages pédagogiques du FF, les derniers soulignent sans ambages les limites de vocabulaires didactiques composés à l'aide de méthodes computatives. On ne s'étonnera donc guère de trouver des chercheurs français parmi ceux qui exigent le centrage sur l'apprenant et par ce biais, le développement d'un autre type de glossaire didactique. A titre d'exemple on citera Richterich et Chancerel (1977), Coste (1978, 1980), Porcher (1980) et Richterich (1980). Il ne surprendra donc pas non plus que le Niveau-Seuil (français) devance les inventaires parallèles destinés à l'apprentissage des autres langues européennes. Les mots-pivots de ce débat : situations communicatives et vocabulaires, définition des besoins langagiers à partir des apprenants, fonctions communicatives - dans le sillage d'Austin (1972)- et minima, spécifications et progressions de l'input, centrage sur les apprenants, problématisation des méthodes lexico-statistiques, abandon d'une croyance quelque peu naïve dans l'utilité de la linguistique computationnelle pour la composition des vocabulaires de base.

Que le FF continuât pourtant, malgré ces critiques, à être un projet qui invitait à développer des projets similaires, est souligné de manière directe par Rivenc (1973) dans son article A l'aube de l'ère des corpus (étude comparée du Français fondamental, de l'Espagnol fondamental et $d u$ Portugais fondamental) ${ }^{7}$. Encore en 1977, A. Giovanni Sciarone publie un Vocabulario fondamentale della lingua italiana dont il écrit, tout à fait dans le sillage du FF: « Il nostro scopo, didattico, è di trovare una lista di parole con un rango (basato sulla frequenza) che sia più o meno indipendente da un determinato contesto " (84). Deux années plus tard, cet auteur articule, dans la revue Die Neueren Sprachen ses réserves vis-à-vis du FF : « La différence fondamentale entre liste de 
fréquence et liste de disponibilité est que la première représente le vocabulaire tel qu'on l'utilise, tandis que la seconde représente (...) nos idées sur la façon dont un vocabulaire est utilisé. » $(1979: 323)$ On comprend donc que les tentatives de l'époque de confectionner des vocabulaires de base pour l'allemand font de multiples références au FF et à la discussion hexagonale esquissée (voir : Kaufmann $1977: 48$ ).

Parfois, cet intérêt semblait provoquer des approches qui, à première vue, paraissent plutôt bizarres. Ainsi lit-on dans la Praxis des neusprachlichen Unterrichts 1953/55 un petit article intitulé 'Das Häufigkeitsprinzip im Sprachunterricht' où l'auteur, T. Marthaler, fait allusion aux recherches canadiennes faites par R. Vinette, auteur de l'Echelle de vocabulaire (éditée par le Centre de Psychologie et de Pédagogie, Montréal 1949). En s'orientant sur cet ouvrage, Marthaler a demandé de son côté à 160 élèves germanophones entre 9 et 14 ans de noter tous les mots qui leur venaient à l'esprit. Ainsi il a obtenu sur 14.078 types 2.802 tokens. La réflexion de l'auteur suit unilatéralement les théories du psychologue Herbart. Marthaler pense que, en s'appuyant sur les mots les plus souvent associés par les apprenants, une méthode de langue parviendrait à provoquer des effets de libération des capacités mémorielles.

La prépondérance du débat allemand autour du FF ne signifie nullement que la théorie ayant trait aux questions de glossaires pédagogiques n'y ait pas été développée (voir Kühn 1990). Déjà, en 1949, G. Nickolaus, auteur du fameux Grund- und Aufbauwortschatz Französisch qui, entre 1963 et 1970 voit 14 ré-éditions, avait composé son Modernes Französisch. Aufgebaut nach der Methode der Worthäufigkeit, 1949 (2 vols.). Pendant quelques années, le $\mathrm{FF}$ à sembler fournir les critères par excellence pour évaluer d'autres vocabulaires de base. A titre d'exemple, nous citons l'article d'I. Christ Ein verbindlicher Grundwortschatz für den Französischunterricht? (1970). Le lecteur y trouve, sur fond de discussion scolaire esquissée, une analyse du 'longseller' de Nickolaus ouvrage-phare de toute une série de petits dictionnaires du même type destinés à toutes les langues scolaires et au-delà, lancés par la maison Klett, leader sur le marché allemand des langues. I. Christ base sa critique sur deux points : le débat français autour du FF et les besoins de l'école allemande. H. Stammerjohann observe qu'elle a mis en relief les écarts entre la liste de Nickolaus et le FF (1983 : 169). En outre, elle a constaté ses faiblesses pour la 'production écrite' telle que la pratique scolaire la demandait des élèves allemands. Deux années plus tard, les lecteurs de Praxis des neusprachlichen Unterrichts liront la riposte de Nickolaus où il dévoile les sources dont il s'était servi pour la confection de son Grund und Aujbauwortschatz (1972:327) :

Das Problem, das sich mir stellte und das ich mit dem Grund-und Aujbauwortschatz zu lösen suchte, war die Synthese zwischen Häufigkeitswortschatz des schriftlichen und denen des mündlichen Sprachgebrauchs. Die Grundlage für die Schriftsprache ist immer noch VAN DER BEKE (sie). Es wurde von James Burton THARP in seinem BASIC French Vocabulary gründlich durchforstet. Diese Ausgabe wurde von mir hauptsächlich benutzt. Die Grundlage für die Redewendungen bildete die Frequenzliste von F. D. CHEYDLEUR, French Idioms List.

Le problème à résoudre concernait donc les écarts de fréquence entre les registres $d u$ parlé et de l'écrit.

Es ist müßig, hier alle sonst in Betracht kommenden Wortlisten anzuführen, da sie alle darin übereinstimmen, daß die ersten 2000 Wörter der VANDER BEKE-Liste als absolute Grundlage anzusehen sind. Daß es sich wirklich bei diesen 2000 Wörtern semantisch um den Grundwortschatz einer Sprache handelt - also nicht nur des Französischen - hat Helen Eaton (...) überzeugend nachgewiesen. Daher war für meinen Grundwortschatz diese Basis von 2000 Wörtern gegeben. 
En 1972, Raasch permettait aux lecteurs germanophones, grâce à son article Neue Wege $z u$ einem Grundwortschatz, de se faire une idée des évolutions françaises. Il s'agit d'une synthèse qui embrasse à la fois des publications francophones et les grands traits du débat mené en Allemagne. L'auteur compare la méthode compilatrice du FF à celles du Französischer Mindestwortschatz et du Grunddeutsch de J. A. Pfeffer (1970) dont Kühn (1990 : 1356) notera la dépendance méthodologique du FF. Ensuite Raasch aborde la problématique épineuse des statistiques lexicales dans le domaine de la langue parlée et explique la notion de la puissance inclusive (coverage) développée par Mackey et Savard (1967) ${ }^{8}$. Comme le FF n'avait jamais vraiment pu satisfaire tous les besoins scolaires de FLE en Allemagne, le romaniste sarrois réitère la critique vis-à-vis de l'utilité des listes lexicométriques :

Auf der einen Seite ist eine Reihe von lexikologischen und lexikometrischen Problemen zu nennen (...). Auf der anderen Seite die spezielleren Probleme: a) Auswahl des Corpus, die Definition von Textsorten und deren Gewichtung, Kriterien für die Gewichtung der Grenzen zwischen den Materialien, die zum angestrebten Grundwortschatz gehören sollen, und jenen, die zu geringe Indices aufweisen. Mathematisch gesehen ist jede Grenzziehung dieser Art willkürlich. Das Heranziehen nicht-statistischer Methoden wird hier unausweichlich. (1972 : 79)

Un peu plus tard, Raasch s'en prend à la croyance trop naïve selon laquelle une connaissance de 2.000 mots permettrait la compréhension de 85 pour cent d'un texte 'normal' (sic), que 2.500 mots de plus entraîneraient une amélioration de 10 pour cent, et que finalement 2.000 mots supplémentaires apporteraient en plus 2 pour cent. Cet argument, attribué à C. Heupel, auteur du Grund und Aufbauwortschatz Spanisch, s'est révélé très efficace sur le marché des vocabulaires de base (voir Heupel 1964). Ceci explique pourquoi il se retrouve dans toutes les listes de ce type de l'époque de la maison Klett (langues-cibles : anglais, français italien, portugais, allemand langue étrangère...). On savait pourtant que les mots rares sont souvent porteurs de l'information-clef pour la compréhension d'un message tandis que les mots fréquents sont sémantiquement assez 'pauvres'. Dans le cadre de cet article, il n'est pas possible de discuter in extenso les arguments exposés par Raasch. Disons rapidement que le chercheur retourne la méthode appliquée pour confectionner le FF en proposant les démarches suivantes : (1) fixation des objectifs pédagogiques, définition des destinataires et analyse du contexte d'apprentissage, (2) compilation des matériaux, (3) description linguistique et analyse statistique.

Deux ans plus tard, Raasch présentera un nouveau projet lexico-didactique devant le public germanophone. Dans Ein neues Français Fondamental? Das Europarat-Projekt Un Niveau Seuil (1978), il montre que les défauts attribués au FF n'y figurent plus. A partir de cette date, les références au FF et aux publications qu'il avait déclenchées se font plus rares. Le FF continue à être cité par contre régulièrement dans les études qui décrivent la qualité des corpus du français parlé. Dans cette fonction, il est aussi mentionné dans la thèse d'état de D. Walz Zur Auswahl, Verteilung, Anordnung und Darstellung des syntaktischen Stoffes für den Französischunterricht an Oberschulen der DDR (Greifswald $1990^{\circ}$ ). Le français n'a commencé à faire vraiment partie des matières scolaires en RDA qu'à partir de 1983, ce qui explique l'absence d'auteurs est-allemands dans le débat du FF. Mais l'invention du Niveau Seuil en 1976 avait déjà ouvert une autre page de l'histoire des minima lexicaux ... européenne cette fois. 


\section{BIBLIOGRAPHIE}

ALI BOUACHA, A. (1987), Vers un niveau 3, Paris : Hachette.

ALI BOUACHA, A. (éd.), (1978), La pédagogie du français langue étrangère, Paris : Hachette.

AUSTIN, J. L. (1959), How To Do Things With Words, Oxford : UP.

BARRERA-VIDAL, A. (1971), Das VHS-Zertifikat fir Spanisch, Frankfurt/M., Pädagogische Arbeitsstelle des Deutschen Volkshochschulverbandes.

BARRERA-VIDAL, A. (1977), «Zur didaktischen Begründung der Sprachminima für den Spanischunterricht» Sprachminima und Abschlussprofile, Hüllen, Raasch, Zapp (éds), pp. 21-28.

BESSE, H. (1979), « Contribution à l'histoire du français fondamental », Le français dans le monde 148, pp. 23-30.

BOLTON, S. et HALM, W. (1979), «L'enseignement des langues aux adultes dans les Universités populaires en RFA », Le français dans le monde 149, pp. 41-53.

CHEYDLEUR, F. D. (1929), French Idiom List. Based on a Count of 1.183.000 Running Words, New York : Macmillan.

CHRIST, I. (1970), «Ein verbindlicher Grundwortschatz für den Französischunterricht? » Praxis des neusprachlichen Unterrichts 17, pp. 32-40.

COSTE, D. (1973), «Français fondamental, enseignement et idéologie, quelques aspects de divers polémiques ", Voix et images du CREDIF, publiées en hommage à la mémoire de G. Gougenheim 18, pp. $4-11$.

COSTE, D. (1978), « Un niveau seuil », La pédagogie du français langue étrangère, Ali Bouacha A, Paris : Hachette, pp. 105-115.

DEBYSER, F. (1970), « L'enseignement du français langue étrangère au niveau 2 », Le français dans le monde 73, pp. 6-14.

EATON, H. S. (1934), Comparative Frequency List. A Study based on the first 1000 words in English, French, German and Spanish Lists, New York : International Auxiliary Language Association.

ESSER, G. (1973), « Zur Definition der Ziele und Inhalte auf Niveau 2 », Die Neueren Sprachen 94, pp. 461-467.

FRANKE, L. (1977), « Abschlußprofile der Sekundarstufe I für Französisch » Sprachminima und Abschlussprofile, Hüllen, Raasch et Zapp (éds), pp. 104-117.

GALISSON, R. (1970), L'apprentissage systématique du vocabulaire. I ; Livre d'exercices II, Paris : Hachette/Larousse.

GALISSON, R. (1971), Inventaire thématique et syntagmatique du français fondamental, Paris : Hachette.

GOUGENHEIM, G. (1958), Dictionnaire fondamental de la langue française (nouv. éd. revue et corrigée), Paris : Didier.

GOUGENHEIM, G. MICHEA, R., RLVENC, P. et SAUVAGEOT, A. (1964), L'élaboration du français fondamental (1er degré, étude sur l'établissement d'un vocabulaire et d'une grammaire de base), Paris : Didier. 
GOUGENHEIM, G., MICHEA, R., RIVENC, P. et SAUVAGEOT, A. (1956), L'élaboration du français élémentaire. Etude sur l'établissement d'un vocabulaire et d'une grammaire de base, Paris : Didier.

GREIVE, A. (1984), «Remarques sur l'histoire du français parlé», Cahiers de l'Institut de Linguistique de Louvain 10, pp. 65-76.

HEUPEL, C. (1964), « Frequenzen und Strukturen im modernen Sprachunterricht », Deutschunterricht für Ausländer 2,3/, pp. 56-63.

HÜLLEN, W., RAASCH, A.ET ZAPP, F. J. (éds.), (1977), Sprachminima und Abschlussprofile, Frankfurt/ M.: Diesterweg.

KAEDING, F. W. (1897), Häufigkeitswörterbuch der deutschen Sprache, Berlin, (Selbstverlag).

KAUFMANN, G. (1977), « Die Gewinnung lexikalischer und grammatischer Minima als linguistisches und didaktisches Problem » Sprachminima und Abschlussprofile, Hüllen, Raasch et Zapp (éds), pp. 48-70.

KÜHN, P. (1990), « Das Grundwortschatzwörterbuch», Wörterbücher. Dictionaries, Dictionnaires. International Encyclopedia of Lexicography, Hausmann F. J., Reichmann O., Wiegand H. E et Zgusta L. (éds.), V, 2/XIII Berlin/New York : de Gruyter, pp. 1353-1364.

LÜBKE, D. (1975), Emploi des mots - Lernwörterbuch Französisch, Dortmund : Lensing. (1987 13e éd.).

LÜBKE, D. (1981), Dictionnaire scolaire du français, München :Langenscheidt.

MACKEY, W. et SAVARD, J.-G. (1967), « The Indice of Coverage: A New Dimension in Lexicometrics ", International Review of Applied Linguistics 2/3, pp. 71-121.

MARTHALER, T. (1953/1955), « Das Häufigkeitsprinzip im Sprachunterricht ». Praxis des neusprachlichen Unterrichts 2/3, pp. 24-25.

MEISSNER, F.-J. (2000), « Kommunikation im Unterricht romanischer Fremdsprachen. Eine historische Skizze », Kommunikationsformen im Wandel der Zeit. Vom mittelalterlichen Heldenepos zum elektronischen Hypertext, Fritz G., Jucker A. (éds.), Tübingen : Niemeyer, pp. 89-119.

MICHEA, R. (1953), « Mots fréquents et mots disponibles. Un aspect de la statistique du langage », Langues Modernes 4, pp. 338-344.

MICHEA, R. (1969), « Répétition et variétés dans l'emploi des mots », Bulletin de la société de linguistique de Paris, fascicule 1, pp. 1-21.

MICHEA, R. (1978), « Les vocabulaires fondamentaux dans l'enseignement des langues ». Le français dans le monde 103, pp. 11-13.

MICHELINI, L. (1988), « Il 'Français fondamental' 30 anni dopo », Scuola e lingue moderne 26/8, pp. 268-278.

MINISTERE DE L'EDUCATION NATIONALE (1959), Français fondamental (1er degré, 2e degré), Paris : Institut Pédagogique National.

MINISTERE DE L'EDUCATION NATIONALE, (1958), Le Français élémentaire. Deutsche Fassung der vom französischen Unterrichtsministerium 1954 herausgegebenen Broschüre über Elementarfranzösisch', Hrsg. vom Institut Français Mainz, Centre de Documentation.

NICKOLAUS, G. (1949), Modernes Französisch. Aufgebaut nach der Methode der Worthäufigkeit, Freiburg/Brsg, Bielefeld, 2 vols.

NICKOLAUS, G. (1963), Grund und Aufbauwortschatz Französisch, Stuttgart : Klett. 
NICKOLAUS, G. (1972), « Noch einmal: 'Ein verbindlicher Grundwortschatz für den Französischunterricht? Stellungnahme zu dem Aufsatz von Christ I. (1970 : 32-40 et 342-349) », Praxis des neusprachlichen Unterrichts 19, pp. 327-328.

OGDEN, CH. K. (1930), Basic English. A General Introduction with Rules and Grammar, London : Keagan Paul.

PFEFFER, J. A. (1970), Grunddeutsch. BASIC (Spoken) German Dictionary, Englewood Cliffs, Prentice Hall.

PORCHER, L. (1980), Interrogations sur les besoins langagiers en contextes scolaires, Strasbourg: Conseil de l'Europe.

RAASCH, A. (1969), Französischer Mindestwortschatz, mit einem Anhang: Grammatische Grundstrukturen, München : Hueber.

RAASCH, A. (1972), « Neue Wege zu einem Grundwortschatz », Praxis des neusprachlichen Unterrichts 19, pp. 235-244.

RAASCH, A. (1978), « Eine neues Français Fondamental! Das Europarat-Projekt 'Un Niveau Seuil' ", Praxis des neusprachlichen Unterrichts 25, pp. 292-301.

RAASCH, A. (1979a), «L'enseignement des langues aux adultes - une notion jeune », Le français dans le monde 149, p. 14.

RAASCH, A. (1979b), « S'auto-évaluer, un néologisme?» Le français dans le monde 149, pp. 63-67.

REISKE, H. (1974), VHS-Italienischunterricht und VHS-Zertifikat Italienisch, Bonn et Frankfurt/M. : Pädagogische Arbeitsstelle des VHS-Verbandes.

RICHTERICH, R., CHANCEREL, J. L. (1977), L'identification des besoins des adultes apprenant une langue étrangère, Paris : Hatier.

RICHTERICH, R. (1980), « L'antidéfinition des besoins langagiers comme pratique pédagogique », Le français dans le monde 149, pp. 54-58.

RIVENC, P. (1973), «A l'aube de l'ère des corpus (étude comparée du Français fondamental, de l'Espagnol fondamental et du Portugais fondamental) », Voix et image du CREDIF 18, Paris : Didier, pp. 12-16.

RlVENC, P. (1979), « Le Français fondamental vingt-cinq ans après », Le français dans le monde 148, pp. 15-22.

ROJOSASTRE, A. J., RVENC, P. et FERRER, A. (1968), Vida y diálogos de España. Primer grado. Método audiovisual realizado bajo los auspicios del Ministerio de Educación y Ciencia de España. Comisaria de Extension Cultural, Paris : Didier (2a ed. 1978).

SAVARD, J.-G. et RICHARDS, J. (1970), Les indices d'utilité du vocabulaire fondamental français, préface par W. F. Mackey, Québec : Presse de l'Université de Laval.

SCHERFER, P. (1969), «Das Français fondamental und seine Verwertbarkeit im fremdsprachlichen Elementarunterricht», Französisch an Volkshochschulen, pp. 6-12.

SCHNEIDER, J. (1937), Der deutsche Wortschatz für niederländische Schüler, Batavia : Noordhoff-Kolff. SCIARONE, A. G. (1977), Vocabolario fondamentale della lingua italiana con una premessa di Giovanni Freddi, Bergamo : Minerva Italica.

SCIARONE, A. G. (1979), « Fréquence et disponibilité », Die Neueren Sprachen 78, pp. 324-331. 
STAMMERJOHANN, H. (1983), Französisch für Lehrer. Linguistische Daten für Studium und Unterricht, München : Hueber.

THARP, J. B. (1934), The Basic French Vocabulary, New York.

VANDER BEKE, G.E. (1929), French Word Book, New York : The MacMillan Company.

VHS-VERBAND, (1977), Das VHS-Zertifikat Spanisch, Frankfurt/M : Pädagogische Arbeitsstelle des VHS-Verbandes.

ZAPP, F. J. et SCHRÖDER K. 1983), Deutsche Lehrpläne für den Fremdsprachenunterricht. 1900-1970. Ein Lesebuch, Augsburg : I\&I.

ZEIDLER, H. (1980), Das «français fondamental» (premier degré). Entstehung, linguistische Analyse und fremdsprachendidaktischer Ort, FrankfurtM. : Peter Lang.

\section{NOTES}

1. Traduit par nous.

2. Ainsi le FE voyait déjà clairement la nécessité d'adapter un corpus lexical didactique aux profils culturels et communicatifs d'autres pays, tout comme le documentera plus tard le Dictionnaire du français fondamental pour l'Afrique publié par J. David (Didier 1974).

3. Rappelons que F. Debyser tentera, en 1970, de développer une didactique propre au niveau 2 ; auquel A. Ali Bouacha ajoutera, en 1987, un niveau 3.

4. Le public adulte se distingue de celui des écoles; d'abord par son âge, ensuite par un intérêt souvent professionnel, son pouvoir d'achat et last but not least, en grande partie, par son ambition de progresser vite et de manière contrôlée dans la langue-cible choisie. Les VHS-Zertifikate répondaient donc en même temps à une réflexion pédagogique et à une demande de la formation continue et du marché de travail, ce qui explique leur succès.

5. Bolton et Halm (1979: 41) : «A l'origine, il y avait une formulation relativement globale des objectifs d'apprentissage, et, en plus, une liste du vocabulaire et des structures grammaticales ; pour le français on a élaboré ces listes en se servant du Français fondamental. »

6. En partie, l'Allemagne devait ces glossaires à l'étranger pour ce qui concerne l'allemand comme langue étrangère (p.ex. Schneider 1937, Michéa 1959).

7. Rojosastre, Rivenc \& Ferrer (1968: 9) : «Durante el verano de 1963, comenzaron en España las actividades de un programa de investigaciones sobre lingüística española que tenía el propósito de recoger y estudiar lo que más tarde hemos llamado 'EL ESPAÑOL FUNDAMENTAL'. (...) Nuestra finalidad era obtener los materiales lingüísticos que supusieran una información suficiente del español hablado, establecida a partir de criterios estadísticos. »

8. "The coverage or covering capacity of an item is the number of things one can say with it. It can be measured by the number of other items which can it displace. » (Mackey $1967: 184$ )

9. Cette thèse n'a pas été publiée. Les observations concernant le FF se trouvent à la p. 9. 
INDEX

Mots-clés : vocabulaire de base, français fondamental, UNESCO, BASIC, enseignement du français aux adultes

Keywords : basic vocabulary, Fundamental French, UNESCO, BASIC English, French teaching for adults

\section{AUTEUR}

FRANZ-JOSEPH MEISSNER

Universität Giessen/Allemagne 